\title{
A capacity approach to the Poincaré inequality and Sobolev imbeddings in variable exponent Sobolev spaces
}

\author{
Petteri Harjulehto and Peter Hästö \\ Department of Mathematics \\ P.O. Box 4 (Yliopistonkatu 5) \\ FIN-00014 University of Helsinki, Finland \\ petteri.harjulehto@helsinki.fi, \\ peter.hasto@helsinki.fi
}

Recibido: 7 de Mayo de 2003

Aceptado: 10 de Septiembre de 2003

\begin{abstract}
We study the Poincaré inequality in Sobolev spaces with variable exponent. Under a rather mild and sharp condition on the exponent $p$ we show that the inequality holds. This condition is satisfied e. g. if the exponent $p$ is continuous in the closure of a convex domain. We also give an essentially sharp condition for the exponent $p$ as to when there exists an imbedding from the Sobolev space to the space of bounded functions.
\end{abstract}

Key words: Sobolev spaces, variable exponent, Poincaré inequality, Sobolev imbedding, continuity

2000 Mathematics Subject Classification: 46E35

\section{Introduction}

There has recently been a surge of interest in Sobolev spaces with variable exponent, cf. [4-7, 9-11, 17, 22]. These spaces, introduced in [17], are the natural generalization of Sobolev spaces to the non-homogeneous situation; they have been used e. g. in modeling electrorheological fluids, see the book of M. Růžička, [22]. Lebesgue and Sobolev spaces with variable exponent share many properties with their classical equivalents, but there is also some crucial differences. For instance the Hardy-Littlewood maximal

The second author was supported financially by the Academy of Finland. 
operator is bounded on $L^{p(\cdot)}$ if the exponent is 0-Hölder continuous (i. e. satisfies (10)) and $1<\operatorname{ess} \inf p \leqslant \operatorname{ess} \sup p<\infty$, [5]. If the exponent is not 0 -Hölder continuous, then the maximal operator need not be bounded on $L^{p(\cdot)},[21]$.

The Poincaré inequality, although of great importance in classical non-linear potential theory (especially in metric spaces) has not been previously studied in the case of variable exponent Sobolev spaces. Our first result, Theorem 2.2, is the following: If $D \subset \mathbb{R}^{n}$ is smooth domain, say a John domain, and the essential supremum of $p$ is less than the Sobolev conjugate of the essential infimum of $p$ then the Poincaré inequality

$$
\left\|u-u_{B}\right\|_{L^{p(\cdot)}(D)} \leqslant C\|\nabla u\|_{L^{p(\cdot)}(D)}
$$

holds for every $u \in W^{1, p(\cdot)}(D)$, where $u_{B}=f u(x) d x$. Here the constant $C$ depends on $n, p, \operatorname{diam}(D)$ and the John constant of $D$. We give an example which shows that the condition for $p$ is sharp even in a ball. It follows from this that if $p$ is continuous in the closure of a convex domain then the Poincaré inequality holds (Corollary 2.7).

In classical theory the constant of the Poincaré inequality is $C \operatorname{diam}(D)$. It is possible to achieve this also for variable exponent Sobolev spaces, as we prove in Corollary 2.10. The price we have to pay is that the exponent $p$ has to be 0 -Hölder continuous.

Sobolev imbeddings in variable exponent Sobolev spaces have been studied by many authors in the case when $p$ is less than the dimension, see [6,9-11]. We give two results in the case when $p$ is greater than the dimension. We prove a result for continuity of the Sobolev functions, namely that every Sobolev function is continuous if the exponent is locally bounded away from the dimension. We show that if a domain satisfies a uniform interior cone condition and $p(x) \geqslant n+f(d(x, \partial G))$ for every $x$ and a certain increasing function $f$ then there exists an imbedding from the variable exponent Sobolev space to $L^{\infty}$. Our condition is essentially sharp.

\section{Notation}

We denote by $\mathbb{R}^{n}$ the Euclidean space of dimension $n \geqslant 2$. For $x \in \mathbb{R}^{n}$ and $r>0$ we denote an open ball with center $x$ and radius $r$ by $B(x, r)$.

Let $A \subset \mathbb{R}^{n}$ and $p: A \rightarrow[1, \infty)$ be a measurable function (called a variable exponent on $A$ ). We define $p_{A}^{+}=\operatorname{ess}_{\sup _{x \in A}} p(x)$ and $p_{A}^{-}=\operatorname{essinf}_{x \in A} p(x)$. If $A=\mathbb{R}^{n}$ we write $p^{+}=p_{\mathbb{R}^{n}}^{+}$and $p^{-}=p_{\mathbb{R}^{n}}^{-}$.

Let $\Omega \subset \mathbb{R}^{n}$ be an open set. We define the generalized Lebesgue space $L^{p(\cdot)}(\Omega)$ to consist of all measurable functions $u: \Omega \rightarrow \mathbb{R}$ such that

$$
\varrho_{p(\cdot)}(\lambda u)=\int_{\Omega}|\lambda u(x)|^{p(x)} d x<\infty
$$

for some $\lambda>0$. The function $\varrho_{p(\cdot)}: L^{p(\cdot)}(\Omega) \rightarrow[0, \infty)$ is called the modular of the space $L^{p(\cdot)}(\Omega)$. One can define a norm, the so-called Luxemburg norm, on this space by the formula $\|u\|_{p(\cdot)}=\inf \left\{\lambda>0: \varrho_{p(\cdot)}(u / \lambda) \leqslant 1\right\}$. Notice that if $p \equiv p_{0}$ then 
$L^{p(\cdot)}(\Omega)$ is the classical Lebesgue space, so there is no danger of confusion with the new notation.

The generalized Sobolev space $W^{1, p(\cdot)}(\Omega)$ is the space of measurable functions $u: \Omega \rightarrow \mathbb{R}$ such that $u$ and the absolute value of the distributional gradient $\nabla u=$ $\left(\partial_{1} u, \ldots, \partial_{n} u\right)$ are in $L^{p(\cdot)}(\Omega)$. The function $\varrho_{1, p(\cdot)}: W^{1, p(\cdot)}(\Omega) \rightarrow[0, \infty)$ is defined as $\varrho_{1, p(\cdot)}(u)=\varrho_{p(\cdot)}(u)+\varrho_{p(\cdot)}(|\nabla u|)$. The norm $\|u\|_{1, p(\cdot)}=\|u\|_{p(\cdot)}+\|\nabla u\|_{p(\cdot)}$ makes $W^{1, p(\cdot)}\left(\mathbb{R}^{n}\right)$ a Banach space.

See [17] for basic properties of variable exponent Lebesgue and Sobolev spaces.

\section{The Poincaré inequality}

In this section we give a relatively mild condition on the exponent for the Poincaré inequality to hold. We also show that this condition is, in a certain sense, the best possible. For Sobolev functions with zero boundary values the Poincaré inequality was given in [10, Lemma 3.1] and considerably generalized in [14].

Recall the following well known Sobolev-Poincaré inequality. By $q^{*}$ we denote the Sobolev conjugate of $q<n, q^{*}=n q /(n-q)$.

Lemma 2.1. Let $D \subset \mathbb{R}^{n}$ be a bounded John domain. Let $1 \leqslant p<n$ and $p \leqslant q \leqslant p^{*}$ be fixed exponents. Then

$$
\left\|u-u_{D}\right\|_{q} \leqslant C(n, p, \lambda)|D|^{1 / n+1 / q-1 / p}\|\nabla u\|_{p}
$$

for all functions $u \in W^{1, p}(D)$, where $\lambda$ is the John constant.

If $p \geqslant n$ and $q<\infty$ then

$$
\left\|u-u_{D}\right\|_{q} \leqslant C(n, q, \lambda)|D|^{1 / n+1 / q-1 / p}\|\nabla u\|_{p}
$$

for all functions $u \in W^{1, p}(D)$.

Proof. The case $p<n$ and $q=p^{*}$ is by B. Bojarski [3, (6.6)]. The case $q<p^{*}$ follows from this by standard arguments: we choose $s \in[1, n)$ such that $s^{*}=q$ (or $s=1$ if $\left.q<1^{*}\right)$. By Hölder's inequality and Bojarski's result we obtain

$$
\begin{aligned}
\left(f_{D}\left|u-u_{D}\right|^{q} d x\right)^{\frac{1}{q}} & \leqslant|D|^{-\frac{1}{s^{*}}}\left(\int_{D}\left|u-u_{D}\right|^{s^{*}} d x\right)^{\frac{1}{s^{*}}} \leqslant C|D|^{-\frac{1}{s^{*}}}\left(\int_{D}|\nabla u|^{s} d x\right)^{\frac{1}{s}} \\
& =C|D|^{\frac{1}{s}-\frac{1}{s^{*}}}\left(f_{D}|\nabla u|^{s} d x\right)^{\frac{1}{s}} \leqslant C|D|\left(f_{D}|\nabla u|^{p} d x\right)^{\frac{1}{p}},
\end{aligned}
$$

which is clearly equivalent to the inequalities in the theorem. 
Theorem 2.2. Let $D \subset \mathbb{R}^{n}$ be a bounded John domain, with constant $\lambda$. If $p_{D}^{+} \leqslant$ $\left(p_{D}^{-}\right)^{*}$ or $p_{D}^{-} \geqslant n$ and $p_{D}^{+}<\infty$ then there exists a constant $C=C\left(n, p_{D}^{-}, p_{D}^{+}, \lambda\right)$ such that

$$
\left\|u-u_{D}\right\|_{p(\cdot)} \leqslant C(1+|D|)^{2}|D|^{\frac{1}{n}+\frac{1}{p_{D}^{+}}-\frac{1}{p_{D}^{-}}}\|\nabla u\|_{p(\cdot)}
$$

for every $u \in W^{1, p(\cdot)}(D)$.

Proof. Assume first that $p_{D}^{+} \leqslant\left(p_{D}^{-}\right)^{*}$. Since $p(x) \leqslant p_{D}^{+} \leqslant\left(p_{D}^{-}\right)^{*}$ we obtain by $[17$, Theorem 2.8] and Lemma 2.1 that

$$
\begin{aligned}
\left\|u-u_{D}\right\|_{p(\cdot)} & \leqslant(1+|D|)\left\|u-u_{D}\right\|_{p_{D}^{+}} \\
& \leqslant C\left(n, p_{D}^{-}, \lambda\right)(1+|D|)|D|^{\frac{1}{n}+\frac{1}{p_{D}^{+}}-\frac{1}{p_{D}^{-}}}\|\nabla u\|_{p_{D}^{-}} \\
& \leqslant C\left(n, p_{D}^{-}, \lambda\right)(1+|D|)^{2}|D|^{\frac{1}{n}+\frac{1}{p_{D}^{+}}-\frac{1}{p_{D}^{-}}}\|\nabla u\|_{p(\cdot) .}
\end{aligned}
$$

The case $p_{D}^{-} \geqslant n$ is similar, the only difference is that the constant in the second inequality in the above chain of inequalities is $C\left(n, p_{D}^{+}, \lambda\right)$.

Remark 2.3. John domains are almost the right class of irregular domains for the classical Sobolev-Poincaré inequality, see [3], [1] and [2, Theorem 4.1].

Previous results on Sobolev imbeddings in the variable exponent setting have been derived in domains whose boundary is locally a graph of a Lipschitz continuous function, see [9-11]. It is therefore of interest to note that every domain, whose boundary is locally the graph of a Lipschitz continuous function, is a John domain, see [19]. In particular every ball is a John domain.

If $D$ is a ball in Theorem 2.2, then the constant in inequality (1) is the classical Sobolev-Poincaré inequality in a ball, see for example [18, Corollary 1.64, p. 38].

The next example shows that if $p_{D}^{-}<n$ and $p_{D}^{+}>\left(p_{D}^{-}\right)^{*}$ then there need not exist a constant $C>0$ such that inequality (1) holds for every $u \in W^{1, p(\cdot)}(D)$.

Recall that the variational capacity for fixed $p, \operatorname{cap}_{p}(E, F ; D)$, is defined for sets $E, F$ and open $D$ by

$$
\operatorname{cap}_{p}(E, F ; D)=\inf _{u \in L(E, F ; D)} \int_{D}|\nabla u|^{p} d x
$$

where $L(E, F ; D)$ is the set of continuous functions $u$ that satisfy $\left.u\right|_{E \cap D}=1,\left.u\right|_{F \cap D}=$ 0 and $|\nabla u| \in L^{p(\cdot)}(D)$. We use the short-hand notation $\operatorname{cap}(E, F)$ for $\operatorname{cap}\left(E, F ; \mathbb{R}^{n}\right)$, similarly for $L(E, F)$. For more information on capacities see [15, Chapter 2] or [20]. The following lemma will be used several times to estimate the gradient of variable exponent functions. 
Lemma 2.4 ([15, Example 2.12, p. 35]). For fixed $p \neq 1, n$, arbitrary $x \in \mathbb{R}^{n}$ and $R>r>0$ we have

$$
\operatorname{cap}_{p}\left(\mathbb{R}^{n} \backslash B(x, R), B(x, r)\right)=\omega_{n-1}\left|\frac{p-n}{p-1}\right|^{p-1}\left|R^{(p-n) /(p-1)}-r^{(p-n) /(p-1)}\right|^{1-p} .
$$

Example 2.5. Our aim is construct a sequence of functions in $B=B(0,1) \subset \mathbb{R}^{2}$ for which the constant in the Poincaré inequality (1) goes to infinity. Let $B_{i}=$ $B\left(2^{-i} e_{1}, \frac{1}{4} 2^{-i}\right) \subset \mathbb{R}^{2}$ and $B_{i}^{\prime}=B\left(2^{-i} e_{1}, \frac{1}{8} 2^{-i^{2}}\right) \subset \mathbb{R}^{2}$ for every $i=1,2, \ldots$ and let $1<p_{1}<2$. Choose a function $u_{i} \in C_{0}^{\infty}\left(B_{i}\right)$ with $\left.u_{i}\right|_{B_{i}^{\prime}}=1$ be such that

$$
\left(2 \operatorname{cap}_{p_{1}}\left(B_{i}^{\prime}, \mathbb{R}^{2} \backslash B_{i}\right)\right)^{\frac{1}{p_{1}}} \geqslant\left\|\nabla u_{i}\right\|_{L^{p_{1}}\left(B_{i}\right)} .
$$

Let $p_{2}>2$ and define $p(x)=p_{1} \chi_{B_{i} \backslash B_{i}^{\prime}}(x)+p_{2} \chi_{B_{i}^{\prime}}(x)$ for $x \in B$ with positive first coordinate. Since $\nabla u_{i}=0$ in $B_{i}^{\prime}$ we obtain

$$
\left\|\nabla u_{i}\right\|_{L^{p(\cdot)}\left(B_{i}\right)}=\left\|\nabla u_{i}\right\|_{L^{p_{1}}\left(B_{i}\right)} .
$$

Let $\tilde{B}_{i}=B\left(-2^{-i} e_{1}, \frac{1}{4} 2^{-i}\right)$. We extend $u_{i}$ to $B$ as an odd function of the first coordinate in $\tilde{B}_{i}$ and by zero elsewhere. We also extend $p$ to $B$ as an even function of the first coordinate. We denote the extensions by $\tilde{u}_{i}$ and $\tilde{p}$. By (2) and (3) we obtain

$$
2^{1+\frac{1}{p_{1}}}\left(\operatorname{cap}_{p_{1}}\left(B_{i}^{\prime}, \mathbb{R}^{2} \backslash B_{i}\right)\right)^{\frac{1}{p_{1}}} \geqslant\left\|\nabla \tilde{u}_{i}\right\|_{L^{\tilde{p}(\cdot)}(B)} .
$$

By Lemma 2.4 this yields

$$
\left\|\nabla \tilde{u}_{i}\right\|_{L^{\tilde{p}(\cdot)}(B)} \leqslant C\left(p_{1}\right)\left|\frac{1}{4} 2^{-i \frac{p_{1}-2}{p_{1}-1}}-\frac{1}{8} 2^{-i^{2} \frac{p_{1}-2}{p_{1}-1}}\right|^{\frac{1-p_{1}}{p_{1}}} .
$$

For large $i$ the right hand side is approximately equal to $C\left(p_{1}\right) 2^{-i^{2} \frac{2-p_{1}}{p_{1}}}$.

Since $\left(\tilde{u}_{i}\right)_{B}=0$, we obtain

$$
\left\|\tilde{u}-\left(\tilde{u}_{i}\right)_{B}\right\|_{L^{\tilde{p}(\cdot)}(B)}=\|\tilde{u}\|_{L^{\tilde{p}(\cdot)}(B)} \geqslant\left|B_{i}^{\prime}\right|^{\frac{1}{p_{2}}} \approx 2^{-i^{2} \frac{2}{p_{2}}} .
$$

By inequalities (4) and (5) we find that

$$
\frac{\left\|\tilde{u}-\left(\tilde{u}_{i}\right)_{B}\right\|_{L^{\tilde{p}(\cdot)}(B)}}{\left\|\nabla \tilde{u}_{i}\right\|_{L^{\tilde{p}(\cdot)}(B)}} \geqslant C\left(p_{1}\right) 2^{i^{2}\left(\frac{2}{p_{1}}-1-\frac{2}{p_{2}}\right)} \rightarrow \infty
$$

as $i \rightarrow \infty$ if $\frac{2}{p_{1}}-1-\frac{2}{p_{2}}>0$, that is, if $p_{2}>\frac{2 p_{1}}{2-p_{1}}=p_{1}^{*}$.

We next show that the condition $p_{D}^{+} \leqslant\left(p_{D}^{-}\right)^{*}$ in Theorem 2.2 can be replaced by a set of local conditions. 
Theorem 2.6. Let $D \subset \mathbb{R}^{n}$ be a bounded John domain. Assume that there exist John domains $G_{i}, i=1, \ldots, j$, so that $G_{i} \subset D$ for every $i, D=\cup_{i=1}^{j} G_{i}$ and either $p_{G_{i}}^{+} \leqslant\left(p_{G_{i}}^{-}\right)^{*}$ or $p_{G_{i}}^{-} \geqslant n$ for every $i$. Then there exists a constant $C>0$ such that

$$
\left\|u-u_{D}\right\|_{p(\cdot)} \leqslant C\|\nabla u\|_{p(\cdot)}
$$

for every $u \in W^{1, p(\cdot)}(D)$. The constant $C$ depends on $n, \operatorname{diam}(D),\left|G_{i}\right|, p$ and the John constants of $D$ and $G_{i}, i=1, \ldots, j$.

Proof. Using the triangle inequality of the norm we obtain

$$
\begin{aligned}
\left\|u-u_{D}\right\|_{L^{p(\cdot)}(D)} & \leqslant \sum_{i=1}^{j}\left\|u-u_{D}\right\|_{L^{p(\cdot)}\left(G_{i}\right)} \\
& \leqslant \sum_{i=1}^{j}\left\|u-u_{G_{i}}\right\|_{L^{p(\cdot)}\left(G_{i}\right)}+\sum_{i=1}^{j}\left\|u_{D}-u_{G_{i}}\right\|_{L^{p(\cdot)}\left(G_{i}\right)} .
\end{aligned}
$$

We estimate the first part of the sum using Theorem 2.2. This yields

$$
\begin{aligned}
\left\|u-u_{G_{i}}\right\|_{L^{p(\cdot)}\left(G_{i}\right)} & \leqslant C\left(n, p_{G_{i}},\left|G_{i}\right|, \lambda_{i}\right)\|\nabla u\|_{L^{p(\cdot)}\left(G_{i}\right)} \\
& \leqslant C\left(n, p_{G_{i}},\left|G_{i}\right|, \lambda_{i}\right)\|\nabla u\|_{L^{p(\cdot)}(D)}
\end{aligned}
$$

for every $i=1, \ldots, j$. Here $\lambda_{i}$ is the John constant of $G_{i}$. We next estimate the second part of the sum in (7) using the classical Poincaré inequality for the third inequality. We obtain

$$
\begin{aligned}
\left\|u_{D}-u_{G_{i}}\right\|_{L^{p(\cdot)}\left(G_{i}\right)} & \leqslant\|1\|_{L^{p(\cdot)}\left(G_{i}\right)} f_{G_{i}}\left|u(x)-u_{D}\right| d x \\
& \leqslant\|1\|_{L^{p(\cdot)}\left(G_{i}\right)}\left|G_{i}\right|^{-1} \int_{D}\left|u(x)-u_{D}\right| d x \\
& \leqslant C(n, \operatorname{diam}(D), \lambda)\left|G_{i}\right|^{-1}\|1\|_{L^{p(\cdot)}\left(G_{i}\right)}\|\nabla u\|_{L^{1}(D)} \\
& \leqslant C(n, \operatorname{diam}(D), \lambda)(1+|D|)\left|G_{i}\right|^{-1}\|1\|_{L^{p(\cdot)}\left(G_{i}\right)}\|\nabla u\|_{L^{p(\cdot)}(D)}
\end{aligned}
$$

for every $i=1, \ldots, j$. Here $\lambda$ is the John constant of $D$. Now inequality (6) follows by inequalities (7), (8) and (9).

Corollary 2.7. Let $D \subset \mathbb{R}^{n}$ be a bounded convex domain and let $p: \bar{D} \rightarrow[1, \infty)$ be a continuous exponent. Then there exists a constant $C>0$ such that

$$
\left\|u-u_{D}\right\|_{p(\cdot)} \leqslant C\|\nabla u\|_{p(\cdot)}
$$

for every $u \in W^{1, p(\cdot)}\left(\mathbb{R}^{n}\right)$. 
Proof. Since $p$ is continuous we find for every $x \in \bar{D}$ a constant $r(x)>0$ such that either

$$
p_{B(x, r(x)) \cap D}^{+} \leqslant\left(p_{B(x, r(x)) \cap D}^{-}\right)^{*} \quad \text { or } \quad p_{B(x, r(x)) \cap D}^{-} \geqslant n .
$$

Since $\bar{D}$ is compact it is possible to find finite covering of $D$ with balls $B(x, r(x))$. It is easy to see that each $B(x, r(x)) \cap D$ is a John domain and hence the corollary follows by Theorem 2.6 .

Sometimes it is useful to have better control over the constant in the Poincaré inequality as the domain $D$ changes than we have in (1). In the fixed exponent case the constant of the Poincaré inequality is $C \operatorname{diam}(D)$. We show that this kind of constant is also possible for variable exponent Sobolev spaces. The price we have to pay for this is that the exponent $p$ has to satisfy a much stronger condition in Theorem 2.8 than in Theorem 2.2; in Theorem 2.2 the exponent $p$ could be discontinuous even in every point, but in Theorem 2.8 the exponent is 0-Hölder continuous.

Theorem 2.8. Let $D \subset \mathbb{R}^{n}$ be a bounded uniform domain. Let $p: D \rightarrow \mathbb{R}$ be such that $1<p_{D}^{-} \leqslant p_{D}^{+}<\infty$. Assume that there exists a constant $C>0$ such that

$$
|p(x)-p(y)| \leqslant \frac{C}{-\log |x-y|}
$$

for every $x, y \in D$ with $|x-y| \leqslant \frac{1}{2}$. Then the inequality

$$
\left\|u-u_{D}\right\|_{p(\cdot)} \leqslant C \operatorname{diam}(D)\left(1+\max \left\{|D|^{1 / p_{D}^{+}-1 / p_{D}^{-}},|D|^{1 / p_{D}^{-}-1 / p_{D}^{+}}\right\}\right)\|\nabla u\|_{p(\cdot)},
$$

holds for every $u \in W^{1, p(\cdot)}(D)$. Here the constant $C$ depends on the dimension $n$, the uniform constant of $D$ and $p$.

Proof. Since $W_{0}^{1, p(\cdot)}(D) \hookrightarrow W^{1,1}(D)$ we obtain as in the proof of [12, Theorem 11] for every $u \in W^{1, p(\cdot)}(D)$ that

$$
|u(x)-u(y)| \leqslant C|x-y|(\mathbb{M} \nabla u(x)+\mathbb{M} \nabla u(y))
$$

for almost every $x, y \in D$. Here $\mathbb{M}$ is the Hardy-Littlewood maximal operator:

$$
\mathbb{M} \nabla u(x)=\sup _{r>0} f_{B(x, r)}|\nabla u(y)| d y,
$$

with the understanding that $\nabla u=0$ outside $D$. The constant $C$ depends on the dimension $n$ and the uniform constants of $D$.

Integrating inequality (12) over $y$ we obtain

$$
\begin{aligned}
\left|u(x)-f_{D} u(y) d y\right| & \leqslant \int_{D}|u(x)-u(y)| d y \\
& \leqslant C \operatorname{diam}(D)\left(\mathbb{M} \nabla u(x)+\int_{D} \mathbb{M} \nabla u(y) d y\right) .
\end{aligned}
$$


By Hölder's inequality [17, Theorem 2.1] this yields

$$
\left|u(x)-u_{D}\right| \leqslant C \operatorname{diam}(D)\left(\mathbb{M} \nabla u(x)+\frac{C(p)\|1\|_{L^{p^{\prime}(\cdot)}(D)}}{|D|}\|\mathbb{M} \nabla u\|_{p(\cdot)}\right) .
$$

Since the previous inequality holds point-wise, it is clear that we have an inequality also for the Lebesgue norms of both sides:

$$
\begin{aligned}
& \left\|u-u_{D}\right\|_{p(\cdot)} \leqslant C \operatorname{diam}(D)\left(\|\mathbb{M} \nabla u\|_{p(\cdot)}+\frac{C}{|D|}\|1\|_{p^{\prime}(\cdot)}\|1\|_{p(\cdot)}\|\mathbb{M} \nabla u\|_{p(\cdot)}\right) \\
& \leqslant C \operatorname{diam}(D)\left(1+|D|^{-1} \max \left\{|D|^{1+1 / p_{D}^{+}-1 / p_{D}^{-}},|D|^{\left.1+1 / p_{D}^{-}-1 / p_{D}^{+}\right\}}\right)\|\mathbb{M} \nabla u\|_{p(\cdot)}\right.
\end{aligned}
$$

By [5, Theorem 3.5] (see also [7, Remark 2.2]) the Hardy-Littlewood maximal operator is bounded, and so we obtain

$$
\left\|u-u_{D}\right\|_{p(\cdot)} \leqslant C \operatorname{diam}(D)\left(1+\max \left\{|D|^{1 / p_{D}^{+}-1 / p_{D}^{-}},|D|^{1 / p_{D}^{-}-1 / p_{D}^{+}}\right\}\right)\|\nabla u\|_{p(\cdot)},
$$

where the constant $C$ depends on the dimension $n$, the uniform constant of $D$ and $p$.

Remark 2.9. We refer to [19] for basic properties of uniform domains: Every uniform domain is a John domain. Every domain, whose boundary is locally a graph of a Lipschitz continuous function, is a uniform domain. In particular if $D$ is a ball then the constant in (11) depends on the dimension $n$ and $p$.

Corollary 2.10. Let $p$ be as in the previous theorem. If $B$ is a ball with $|B| \leqslant 1$ then

$$
\left\|u-u_{B}\right\|_{p(\cdot)} \leqslant C \operatorname{diam}(B)\|\nabla u\|_{p(\cdot),}
$$

where the constant $C$ does not depend on $B$.

Proof. Since $|B| \leqslant 1$ we have

$$
\max \left\{|B|^{1 / p_{B}^{+}-1 / p_{B}^{-}},|B|^{1 / p_{B}^{-}-1 / p_{B}^{+}}\right\}=|B|^{1 / p_{B}^{+}-1 / p_{B}^{-}} .
$$

Since $p$ is 0 -Hölder continuous, (10), we obtain by [5, Lemma 3.2] that there exists a constant $C>0$, depending only on the dimension $n$ and the constant in (10), such that $|B|^{1 / p_{B}^{+}-1 / p_{B}^{-}} \leqslant C$ for every ball $B$. Hence $|B| \leqslant 1$ implies that the constant in (11) is less than $C \operatorname{diam}(B)$. 


\section{Continuity}

The functions in the classical Sobolev space $W^{1, p}$ are continuous if $p>n$. In this section we consider when functions in variable exponent Sobolev space are continuous.

Theorem 3.1. Suppose that $p>n$ is locally bounded away from $n$ in $D$. Then $W^{1, p(\cdot)}(D) \subset C(D)$.

Proof. Let $x \in D$ and consider the ball $B=B(x, \delta(x) / 2)$. Define $q=\operatorname{essinf}_{y \in B} p(y)$. Then, by [17, Theorem 2.8],

$$
W^{1, p(\cdot)}(B) \hookrightarrow W^{1, q}(B) \subset C(B) .
$$

Therefore every function in $W^{1, p(\cdot)}(D)$ is continuous at $x$, and since $x$ was arbitrary, the claim follows.

The following corollary is immediate.

Corollary 3.2. Suppose that $p$ is continuous in D. Then $W^{1, p(\cdot)}(D) \subset C(D)$ if $p(x)>n$ for every $x \in D$.

We next use a classical example to show that the assumption that $p$ is locally bounded away from $n$ in $D$ is not superfluous when $p$ is not continuous.

Example 3.3. Let $B=B(0,1 / 16), \varepsilon>0$ and suppose that

$$
p(x) \leqslant \bar{p}(|x|)=n+(n-1-\varepsilon) \frac{\log _{2} \log _{2}(1 /|x|)}{\log _{2}(1 /|x|)}
$$

for $x \in B \backslash\{0\}$ and $p(0)>n$. We show that then $W^{1, p(\cdot)}(B) \not \subset C(B)$.

Define $u(x)=\cos \left(\log _{2}\left|\log _{2}\right| x||\right)$ for $x \in B \backslash\{0\}$ and $u(0)=0$. Clearly $u$ is not continuous at the origin. So we have to show that $u \in W^{1, p(\cdot)}(B)$. It is clear that $u$ has partial derivatives, except at the origin.

Since $u$ is bounded it follows that $u \in L^{p(\cdot)}(B)$. We next estimate the gradient:

$$
|\nabla u(x)|=\left|\sin \left(\log _{2}\left|\log _{2}\right| x||\right) \cdot \frac{1}{|x| \log _{2}|x|}\right| \leqslant\left|\frac{1}{|x| \log _{2}|x|}\right| .
$$

We therefore find that

$$
\begin{aligned}
\int_{B}|\nabla u(x)|^{p(x)} d x & \leqslant \int_{B} \frac{d x}{\left(|x|\left|\log _{2}\right| x||\right)^{p(x)}} \\
& =\omega_{n-1} \int_{0}^{1 / 16} \frac{r^{n-1} d r}{\left(r\left|\log _{2} r\right|\right)^{\bar{p}(r)}} \\
& =\omega_{n-1} \sum_{i=5}^{\infty} \int_{2^{-i-1}}^{2^{i}} \frac{r^{n-1} d r}{\left(r\left|\log _{2} r\right|\right)^{\bar{p}(r)}}
\end{aligned}
$$


Since $1 /\left(r\left|\log _{2} r\right|\right)>1$ we may increase the exponent $\bar{p}$ for an upper bound. In the annulus $B\left(0,2^{-i}\right) \backslash B\left(0,2^{-i-1}\right)$ we have $i \leqslant \log _{2}(1 /|x|) \leqslant i+1$. Since $y \rightarrow \log _{2}(y) / y$ is decreasing we find that

$$
\bar{p}(x) \leqslant n+(n-1-\varepsilon) \frac{\log _{2} i}{i}
$$

in the same annulus. We can therefore continue our previous estimate by

$$
\begin{aligned}
\int_{B}|\nabla u(x)|^{p(x)} d x & \leqslant \sum_{i=5}^{\infty} \int_{2^{-i-1}}^{2^{-i}} \frac{r^{n-1} d r}{\left(r\left|\log _{2} r\right|\right)^{n+(n-1-\varepsilon) \log _{2}(i) / i}} \\
& \leqslant C \sum_{i=5}^{\infty} \int_{2^{-i-1}}^{2^{-i}} \frac{2^{-i(n-1)} d r}{\left(i 2^{-i}\right)^{n+(n-1-\varepsilon) \log _{2}(i) / i}} \\
& =C \sum_{i=5}^{\infty} 2^{(n-1-\varepsilon) \log _{2}(i)} i^{-n-(n-1-\varepsilon) \log _{2}(i) / i} \\
& =C \sum_{i=5}^{\infty} i^{-1-\varepsilon} i^{-(n-1-\varepsilon) \log _{2}(i) / i} \leqslant C \sum_{i=5}^{\infty} i^{-1-\varepsilon}<\infty
\end{aligned}
$$

\section{Sobolev imbedding theorems}

We start by introducing a relative variational $p(\cdot)$-pseudocapacity, and proving some basic properties for it. This capacity is quite similar to the Sobolev $p(\cdot)$-capacity studied by P. Harjulehto, P. Hästö, M. Koskenoja and S. Varonen in [13].

Let $F, E \subset \mathbb{R}^{n}$ be closed disjoint sets and $D$ be a domain in $\mathbb{R}^{n}$. The variational $p(\cdot)$-pseudocapacity is defined as

$$
\psi_{p(\cdot)}(F, E ; D)=\inf _{u \in L(F, E ; D)}\|\nabla u\|_{L^{p(\cdot)}(D)}
$$

where $L(F, E ; D)$ is as before (see Section 2). For $L(F, E ; D)=\emptyset$ we define $\psi_{p(\cdot)}(F, E ; D)=\infty$. We write $L(E, x ; D)$ for $L(F,\{x\} ; D)$ etc.

Remark 4.1. Including $C(D)$ in the definition of the capacity is somewhat strange in this context, since we do not, in general, know whether continuous functions are dense in $W^{1, p(\cdot)}(D)$, but see [8]. However, since we are interested in the case when $p>n$, the assumption makes sense, by Theorem 3.1.

The reason for calling the function $\psi_{p(\cdot)}(F, E ; D)$ a pseudocapacity is that it is defined as a capacity but using the norm instead of the modular. This corresponds to introducing an exponent $1 / p$ to the capacity in the fixed exponent case. Because of this we cannot expect the pseudocapacity to have all the usual properties of a capacity. It nevertheless has many of them: 
Theorem 4.2. Let $F, E \subset \mathbb{R}^{n}$ be closed sets and $D$ be a domain in $\mathbb{R}^{n}$. Then the set function $(F, E) \mapsto \psi_{p(\cdot)}(F, E ; D)$ has the following properties:

(i) $\psi_{p(\cdot)}(\emptyset, E ; D)=0$.

(ii) $\psi_{p(\cdot)}(F, E ; D)=\psi_{p(\cdot)}(E, F ; D)$.

(iii) Outer regularity, i. e. $\psi_{p(\cdot)}\left(F, E_{1} ; D\right) \leqslant \psi_{p(\cdot)}\left(F, E_{2} ; D\right)$.

(iv) If $E$ is a subset of $\mathbb{R}^{n}$, then

$$
\psi_{p(\cdot)}(F, E ; D)=\inf _{\substack{E \subset U \\ U \text { open }}} \psi_{p(\cdot)}(F, U ; D) .
$$

(v) If $K_{1} \supset K_{2} \supset \ldots$ are compact, then

$$
\lim _{i \rightarrow \infty} \psi_{p(\cdot)}\left(F, K_{i} ; D\right)=\psi_{p(\cdot)}\left(F, \bigcap_{i=1}^{\infty} K_{i} ; D\right) .
$$

(vi) Suppose that $p>n$ is locally bounded away from $n$. If $E_{i} \subset \mathbb{R}^{n}$ for every $i=1,2, \ldots$, then

$$
\psi_{p(\cdot)}\left(F, \bigcup_{i=1}^{\infty} E_{i} ; D\right) \leq \sum_{i=1}^{\infty} \psi_{p(\cdot)}\left(F, E_{i} ; D\right) .
$$

Proof. Assertion (i) is clear since we may use a constant function. Assertion (ii) is clear since if $u \in L(F, E ; D)$ then $1-u \in L(E, F ; D)$. Assertion (iii) follows since $L\left(F, E_{2} ; D\right) \subset L\left(F, E_{1} ; D\right)$.

Next we prove (iv). It is clear that

$$
\psi_{p(\cdot)}(F, E ; D) \leqslant \inf _{\substack{E \subset U \\ U \text { open }}} \psi_{p(\cdot)}(F, U ; D) .
$$

Let $\varepsilon>0$. Assume that $u \in L(F, E ; D)$ is such that

$$
\|\nabla u\|_{p(\cdot)} \leqslant \psi_{p(\cdot)}(F, E ; D)+\varepsilon .
$$

Since $u$ is continuous, $\{u>1-\varepsilon\}$ is an open set containing $E$. Hence we obtain

$$
\begin{aligned}
\inf _{\substack{E \subset U \\
U \text { open }}} \psi_{p(\cdot)}(F, U ; D) & \leqslant \psi_{p(\cdot)}(F,\{u>1-\varepsilon\} ; D) \\
& \leqslant\left\|\nabla \min \left\{\frac{u}{1-\varepsilon}, 1\right\}\right\|_{p(\cdot)} \leq(1-\varepsilon)^{-1}\|\nabla u\|_{p(\cdot)} \\
& \leqslant(1-\varepsilon)^{-1} \psi_{p(\cdot)}(F, E ; D)+\frac{\varepsilon}{1-\varepsilon} .
\end{aligned}
$$


Letting $\varepsilon \rightarrow 0$ yields assertion (iv).

We then prove $(\mathrm{v})$. It is clear that

$$
\psi_{p(\cdot)}\left(F, \cap_{i=1}^{\infty} K_{i} ; D\right) \leqslant \lim _{i \rightarrow \infty} \psi_{p(\cdot)}\left(F, K_{i} ; D\right)
$$

Let $\varepsilon>0$. Assume that $u \in L\left(F, \cap_{i=1}^{\infty} K_{i} ; D\right)$ is such that

$$
\|\nabla u\|_{p(\cdot)} \leqslant \psi_{p(\cdot)}\left(F, \cap_{i=1}^{\infty} K_{i} ; D\right)+\varepsilon .
$$

When $i$ is large the set $K_{i}$ lies in the closed set $\{u \geqslant 1-\varepsilon\}$; therefore

$$
\begin{aligned}
\lim _{i \rightarrow \infty} \psi_{p(\cdot)}\left(F, K_{i} ; D\right) & \leqslant \psi_{p(\cdot)}(F,\{u \geqslant 1-\varepsilon\} ; D) \\
& \leqslant\left\|\nabla \min \left\{\frac{u}{1-\varepsilon}, 1\right\}\right\|_{p(\cdot)} \leq(1-\varepsilon)^{-1}\|\nabla u\|_{p(\cdot)} \\
& \leqslant(1-\varepsilon)^{-1} \psi_{p(\cdot)}\left(F, \cap_{i=1}^{\infty} K_{i} ; D\right)+\frac{\varepsilon}{1-\varepsilon}
\end{aligned}
$$

Letting $\varepsilon \rightarrow 0$ yields assertion (v).

To prove (vi) let $\varepsilon>0$ and choose functions $u_{i} \in L\left(F, E_{i} ; D\right)$ such that

$$
\left\|\nabla u_{i}\right\|_{p(\cdot)} \leqslant \psi_{p(\cdot)}\left(F, E_{i} ; D\right)+\varepsilon / 2^{i}
$$

for $i=1, \ldots$ Let $v_{i}=u_{1}+\ldots+u_{i}$. Then $\left(v_{i}\right)$ is a Cauchy sequence, and so it converges to a function $v \in W^{1, p(\cdot)}(D)$. Define $\tilde{v}(x)=\min \{v(x), 1\}$, so that $|\tilde{v}| \in L^{p(\cdot)}(D)$ by [13, Theorem 2.2]. It is clear that $\left.\tilde{v}\right|_{F \cap D}=0$ and $\left.\tilde{v}\right|_{E \cap D}=1$, where $E=\cup E_{i}$. Since $p>n$ is locally bounded away from $n$, it follows from Theorem 3.1 that every function in $W^{1, p(\cdot)}(D)$ is continuous, and so we have $\tilde{v} \in L\left(F, \bigcup E_{i} ; D\right)$, from which the claim easily follows, since

$$
\|\nabla \tilde{v}\|_{p(\cdot)} \leqslant \sum_{i=1}^{\infty}\left\|\nabla u_{i}\right\|_{p(\cdot)} \leqslant \sum_{i=1}^{\infty} \psi_{p(\cdot)}\left(F, E_{i} ; D\right)+\varepsilon .
$$

Using the pseudocapacity we can start our study of Sobolev-type imbeddings. The following result is the direct generalization of $[20,5.1 .1$, Theorem 1].

Theorem 4.3. If $p^{+}<\infty$, then the following two conditions are equivalent:

(i) $W^{1, p(\cdot)}(D) \cap C(D) \hookrightarrow L^{\infty}(D)$.

(ii) There exist $r, k>0$ such that $\psi_{p(\cdot)}(\bar{D} \backslash B(x, r), x ; D) \geqslant k$ for every $x \in D$.

Proof. Suppose that (2) holds, with constants $r, k>0$. Let $u \in W^{1, p(\cdot)}(D) \cap C(D)$ and let $y \in D$ be a point with $u(y) \neq 0$. Fix a function $\eta \in C_{0}^{\infty}(B(0,1))$ with $0 \leqslant \eta \leqslant 1$ and $\eta(0)=1$. Define $v(x)=\eta((x-y) / r) u(x) / u(y)$. It is clear that $v \in W^{1, p(\cdot)}(D)$ 
and since $v(y)=1$ and $v(x)=0$ for $x \notin B(y, r)$ we see that $v \in L(\bar{D} \backslash B(y, r), y ; D)$. It follows that

$$
k \leqslant \psi_{p(\cdot)}(\bar{D} \backslash B(y, r), y ; D) \leqslant\|\nabla v\|_{p(\cdot)} .
$$

Then we calculate that

$$
\begin{aligned}
k|u(y)| & \leqslant\|\nabla(u(x) \eta((x-y) / r))\|_{p(x)} \\
& \leqslant \sup _{x \in D} \eta(x)\|\nabla u\|_{p(\cdot)}+\frac{1}{r} \sup _{x \in D} \nabla \eta(x)\|u\|_{p(\cdot)} \\
& \leqslant \max \left\{\sup _{x \in D} \eta(x), \frac{1}{r} \sup _{x \in D} \nabla \eta(x)\right\}\|u\|_{1, p(\cdot)},
\end{aligned}
$$

so that $|u(y)|$ is bounded by a constant independent of $y$.

Suppose conversely that (1) holds and let $C$ be a constant such that $\|u\|_{\infty} \leqslant$ $C\|u\|_{1, p(\cdot)}$ for all $u \in W^{1, p(\cdot)}(D)$. For functions in $v \in L(\bar{D} \backslash B(x, r), x ; D)$ this gives

$$
1=\|v\|_{\infty} \leqslant C\|v\|_{1, p(\cdot)} \leqslant C\left(\left\|\chi_{B(x, r)}\right\|_{p(\cdot)}+\|\nabla u\|_{p(\cdot)}\right) .
$$

Since $p^{+}<\infty$ we can choose $r$ small enough that $\left\|\chi_{B(x, r)}\right\|_{p(\cdot)} \leqslant 1 /(2 C)$. For such $r$ we have $\|\nabla u\|_{p(\cdot)} \geqslant 1 /(2 C)$. It follows that

$$
\psi_{p(\cdot)}(\bar{D} \backslash B(x, r), x ; D)=\inf _{u \in L(\bar{D} \backslash B(x, r), x ; D)}\|\nabla u\|_{p(\cdot)} \geqslant 1 /(2 C)
$$

for the same $r$.

Remark 4.4. Since we do not know whether $C^{\infty}(D)$ is dense in $W^{1, p(\cdot)}(D)$ we have only proved the theorem for continuous functions in $W^{1, p(\cdot)}(D)$. If $p$ is such that $C(D)$ is dense in $W^{1, p(\cdot)}(D)$, for instance if $p$ is locally bounded above $n$, then we may replace condition (1) by $W^{1, p(\cdot)}(D) \hookrightarrow L^{\infty}(D)$.

Define $D=B(1 / 16) \backslash\{0\}$ and let $p$ be as in Example 3.3. Then the standard example $u(x)=\log |\log (x)|$ shows that $W^{1, p(\cdot)}(D) \hookrightarrow L^{\infty}$, the calculations being as in the theorem. We next show that the exponent $p$ from the theorem is almost as good as possible. We need the following lemma.

Lemma 4.5. Let $\left\{a_{i}\right\}$ be a partition of unity and $k>m-1$. Then

$$
\sum_{i=0}^{\infty} a_{i}^{m} i^{k} \geqslant\left(\sum_{i=0}^{\infty} i^{-k /(m-1)}\right)^{1-m} .
$$

Proof. Fix an integer $i$ and consider the function

$$
a \mapsto\left(a_{i}+a\right)^{m} i^{k}+\left(a_{i+1}-a\right)^{m}(i+1)^{k},
$$


for $-a_{i}<a<a_{i+1}$. We find that this function has a minimum at $a=0$ if and only if

$$
\left(\frac{a_{i}}{a_{i+1}}\right)^{m-1}=\left(\frac{i+1}{i}\right)^{k}
$$

Let $\left\{a_{i}\right\}$ be a minimal sequence, so that (13) holds for every $i \geqslant 0$. This partition is given by $a_{i}=i^{-k /(m-1)} a_{0}$ for $i>0$ and $a_{0}=\left(\sum i^{-k /(m-1)}\right)^{-1}$ and so we easily calculate the lower bound as given in the lemma.

We next give a simple sufficient condition for the imbedding $W^{1, p(\cdot)}(D) \hookrightarrow L^{\infty}(D)$ to hold in a regular domain:

Theorem 4.6. Suppose that $D$ satisfies a uniform interior cone condition. If $p^{+}<\infty$ and

$$
p(x) \geqslant n+(n-1+\varepsilon) \frac{\log _{2} \log _{2}(c / \delta(x))}{\log _{2}(c / \delta(x))}
$$

for some fixed $0<\varepsilon<n-1$ and constant $c>0$ then $W^{1, p(\cdot)}(D) \hookrightarrow L^{\infty}(D)$. Here $\delta(x)$ denotes the distance of $x$ from the boundary of $D$

Proof. Note first that the claim trivially holds in compact subsets of $D$ which satisfy the cone condition, since $p$ is bounded away from $n$ in such sets. Therefore it suffices to prove the claim for $\delta(x)$ less than some constant.

By the uniform interior cone condition there exist real values $0<\alpha<\pi / 2$ and $r>0$ and a unit vector field $v_{x}$ such that for every $x \in D$ the cone

$$
C_{x}=\left\{y \in B(x, r):\left\langle x-y, v_{x}\right\rangle>|x-y| \cos \alpha\right\}
$$

lies completely in $D$, where $\langle\cdot, \cdot\rangle$ denotes the usual inner product.

Fix $z \in D$. Consider the cone

$$
C=\left\{y \in B(z, r / 2):\left\langle z-y, v_{z}\right\rangle>|z-y| \cos (\alpha / 3)\right\}
$$

and, for $i=2,3, \ldots$, the annuli

$$
A_{i}=\left(B\left(z, 2^{-i+1} r\right) \backslash B\left(z, 2^{-i} r\right)\right) \cap C .
$$

To simplify notation let us assume that $z=0, r=1$ and $v_{z}=e_{1}$; the proof in the general case is essentially identical. Since $A_{i} \subset C \subset D$ we have $d\left(A_{i}, \partial D\right) \geqslant$ $d\left(A_{i}, \partial C\right)$. We can estimate the latter distance as shown in Figure 1. This gives $d\left(A_{i}, \partial D\right) \geqslant 2^{-i} \sin (\alpha / 3)$ so that

$$
p(x) \geqslant n+(n-1+\varepsilon) \frac{\log _{2}(i+c)}{i+c}
$$




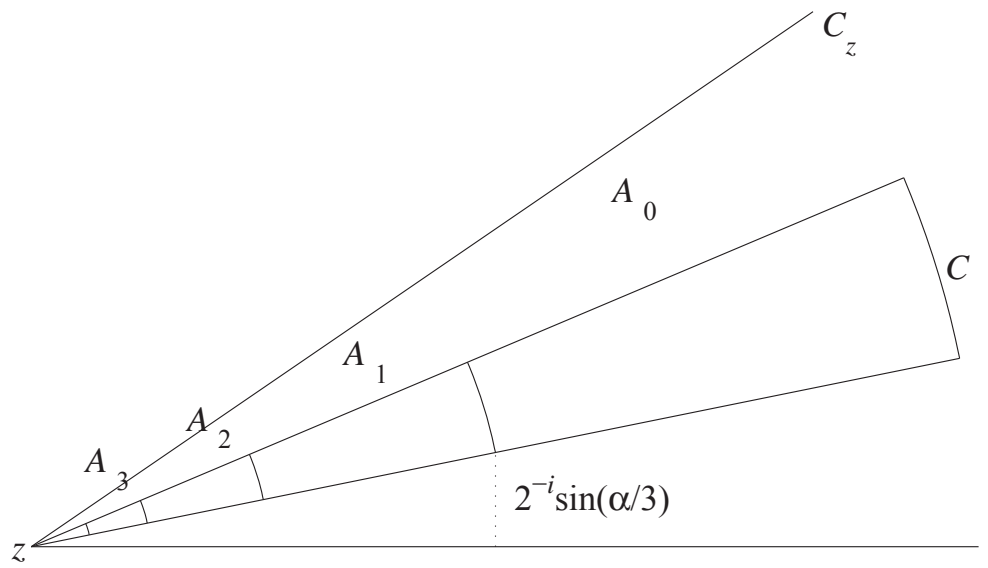

Figure 1: The cone $C$ and the distance to the boundary

for $x \in A_{i}$ and some $c$ depending on $\alpha$. Let us define $q_{i}=n+(n-1+\varepsilon) \frac{\log _{2}(i+c)}{i+c}$ and a new variable exponent by

$$
q(x)= \begin{cases}q_{i} & \text { if } x \in A_{i} \text { for some } i \\ p(x) & \text { otherwise }\end{cases}
$$

By Theorem 4.3 we know that it suffices to find a lower bound for $\|\nabla u\|_{1, p(\cdot)}$ with $u \in L(\bar{D} \backslash B(0, r), 0 ; D)$ since, by Theorem 3.1, $W^{1, p(\cdot)}(D) \subset C(D)$. Since $\|u\|_{1, p(\cdot)} \geqslant$ $c\|u\|_{1, q(\cdot)}$, we see that it suffices to estimate $\psi_{q(\cdot)}(\bar{D} \backslash B(0, R), 0 ; B(0, R) \cap D)$ for small $R$ in order to prove the theorem. Moreover, by monotony, we need only consider $\psi_{q(\cdot)}(\bar{D} \backslash B(0, R), 0 ; B(0, R) \cap C)$. For every function $u \in W^{1, q(\cdot)}(C)$ we have

$$
\|u\|_{1, q(\cdot)} \geqslant \min \left\{1, \varrho_{1, q(\cdot)}(u)\right\}
$$

by $\left[17\right.$, Theorem 2.8]. Therefore we see that it suffices to show that $\varrho_{1, q(\cdot)}(u)>c$ for every $u \in L(\bar{D} \backslash B(0, R), 0 ; B(0, R) \cap C)$ in order to get $\psi_{q(\cdot)}(\bar{D} \backslash B(0, R), 0 ; B(0, R) \cap$ $C) \geqslant \min \{1, c\}>0$, which will complete the proof.

It is clear that $|\nabla u| \geqslant|\partial u / \partial r|$, the radial component of the gradient, so that

$$
\int_{A_{i}}|\nabla u|^{q_{i}} d x \geq \int_{A_{i}}\left|\frac{\partial u}{\partial r}\right|^{q_{i}} d x .
$$

It is then easy to see that the function minimizing the sum over the integrals should depend only on the distance from the origin, not on the direction. For such a function let us denote the value at any point of distance $2^{-i}$ from the origin by $v_{i}$. 
Consider then a function $v$ which equals $v_{i-1}$ on $S\left(0,2^{-i+1}\right)$ and $v_{i}$ on $S\left(0,2^{-i}\right)$. Using Lemma 2.4 we find that

$$
\begin{aligned}
\int_{A_{i}}|\nabla v|^{q_{i}} d x & \geqslant\left(v_{i-1}-v_{i}\right)^{q_{i}} \operatorname{cap}_{q_{i}}\left(\mathbb{R}^{n} \backslash B\left(0,2^{-i+1}\right), B\left(0,2^{-i}\right)\right) \\
& =\left(v_{i-1}-v_{i}\right)^{q_{i}} \omega_{n-1}\left(\frac{q_{i}-n}{q_{i}-1}\right)^{q_{i}-1}\left(2^{\left(q_{i}-n\right) /\left(q_{i}-1\right)}-1\right)^{1-q_{i}} 2^{i\left(q_{i}-n\right)} \\
& \geqslant c\left(v_{i-1}-v_{i}\right)^{q_{i}} 2^{i\left(q_{i}-n\right)},
\end{aligned}
$$

where the constant $c$ does not depend on $q_{i}$. It follows that

$$
\varrho_{1, q(\cdot)}(v) \geqslant \sum_{i=2}^{\infty} \int_{A_{i}}|\nabla u|^{q_{i}} d x \geqslant c \sum_{i=2}^{\infty}\left(v_{i-1}-v_{i}\right)^{q_{i}} 2^{i\left(q_{i}-n\right)} .
$$

Since the lower bound depends only on the $v_{i}$, we see that

$$
\inf _{u \in L} \varrho_{1, q(\cdot)}(u) \geqslant c \inf _{\left\{v_{i}\right\}} \sum_{i=2}^{\infty}\left(v_{i-1}-v_{i}\right)^{q_{i}} 2^{i\left(q_{i}-n\right)},
$$

where the second infimum is over sequences $\left\{v_{i}\right\}$ with $v_{i} \leqslant v_{i-1}, v_{0}=1$ and $\lim _{i \rightarrow \infty} v_{i}=0$. Let us set $a_{i}=v_{i-1}-v_{i}$ so that $a_{i} \geqslant 0$ and $\sum a_{i}=1$. Then we need to estimate

$$
\inf _{\left\{a_{i}\right\}} \sum_{i=2}^{\infty} a_{i}^{q_{i}} 2^{i\left(q_{i}-n\right)},
$$

with the infimum over partitions of unity $\left\{a_{i}\right\}$. Let $N$ be such that

$$
\frac{\varepsilon}{3} \geqslant q_{i}-n=(n-1+\varepsilon) \frac{\log _{2}(i+c)}{i+c} \geqslant(n-1+\varepsilon / 2) \frac{\log _{2}(i)}{i}
$$

for $i \geq N$. Note that such an $N$ can be chosen independent of $z$. Since $a_{i} \leqslant 1$ we have $a_{i}^{q_{i}} \geqslant a_{i}^{n+\varepsilon / 3}$ for such terms. Then we find that

$$
\inf _{\left\{a_{i}\right\}} \sum_{i=2}^{\infty} a_{i}^{q_{i}} 2^{i\left(q_{i}-n\right)} \geqslant \inf _{\left\{a_{i}\right\}} \sum_{i=2}^{N-1} a_{i}^{q_{i}} 2^{i\left(q_{i}-n\right)}+\sum_{i=N}^{\infty} a_{i}^{n+\varepsilon / 3} i^{n-1+\varepsilon / 2} .
$$

The first sum on the left-hand-side is finite, hence

$$
\sum_{i=2}^{N-1} a_{i}^{q_{i}} 2^{i\left(q_{i}-n\right)} \geqslant \sum_{i=2}^{N-1} a_{i}^{q} \geqslant N^{1-q}\left(\sum_{i=2}^{N-1} a_{i}\right)^{q},
$$

where $q=\max _{2 \leqslant i \leqslant N-1} q_{i}$. It follows from Lemma 4.5 that

$$
\sum_{i=N}^{\infty} a_{i}^{n+\varepsilon / 3} i^{n-1+\varepsilon / 2} \geqslant c\left(\sum_{i=N}^{\infty} a_{i}\right)^{n+\varepsilon / 3} .
$$


Combining these estimates we see that

$$
\inf _{\left\{a_{i}\right\}} \sum_{i=2}^{\infty} a_{i}^{q_{i}} 2^{i\left(q_{i}-n\right)} \geqslant N^{1-q}\left(\sum_{i=2}^{N-1} a_{i}\right)^{q}+c\left(\sum_{i=N}^{\infty} a_{i}\right)^{n+\varepsilon / 3}
$$

is uniformly bounded from below by a positive constant, since the sum of the $a_{i}$ 's is 1 . We have thus shown that the condition of Theorem 4.3 holds, which concludes the proof.

\section{References}

[1] S. M. Buckley and P. Koskela, Sobolev-Poincaré implies John, Math. Res. Lett. 2 (1995), 577593.

[2] _ Criteria for imbeddings of Sobolev-Poincaré type, Internat. Math. Res. Notices (1996), 881-901.

[3] B. Bojarski, Remarks on Sobolev imbedding inequalities, Complex Analysis, Joensuu 1987, Lecture Notes in Math., vol. 1351, Springer, Berlin, 1988, pp. 52-68.

[4] D. Cruz-Uribe, A. Fiorenza, and C. J. Neugebauer, The maximal function on variable $L^{p}$ spaces, Ann. Acad. Sci. Fenn. Math. 28 (2003), 223-238.

[5] L. Diening, Maximal function on generalized Lebesgue spaces $L^{p(\cdot)}$, Math. Inequal. Appl. (to appear).

[6] __ Riesz Potential and Sobolev Embeddings of generalized Lebesgue and Sobolev Spaces $\overline{L^{p(\cdot)}}$ and $W^{k, p(\cdot)}$, Math. Nachr. (to appear).

[7] L. Diening and M. Růžička, Calderón-Zygmund operators on generalized Lebesgue spaces $L^{p(\cdot)}$ and problems related to fluid dynamics, J. Reine Angew. Math. 563 (2003), 197-220.

[8] D. E. Edmunds and J. Rákosník, Density of smooth functions in $W^{k, p(x)}(\Omega)$, Proc. Roy. Soc. London Ser. A 437 (1992), 229-236.

[9] _ Sobolev embeddings with variable exponent, Studia Math. 143 (2000), 267-293.

[10] _ Sobolev embeddings with variable exponent. II, Math. Nachr. 246/247 (2002), 53-67.

[11] X. Fan, J. Shen, and D. Zhao, Sobolev embedding theorems for spaces $W^{k, p(x)}(\Omega)$, J. Math. Anal. Appl. 262 (2001), 749-760.

[12] P. Hajłasz and O. Martio, Traces of Sobolev functions on fractal type sets and characterization of extension domains, J. Funct. Anal. 143 (1997), 221-246.

[13] P. Harjulehto, P. Hästö, M. Koskenoja, and S. Varonen, Sobolev capacity on the space $W^{1, p(\cdot)}\left(\mathbb{R}^{n}\right)$, J. Funct. Spaces Appl. 1 (2003), 17-33.

[14] _ Dirichlet energy integral and Sobolev spaces with zero boundary values (preprint. Available in http://www.math.helsinki.fi/analysis/varsobgroup).

[15] J. Heinonen, T. Kilpeläinen, and O. Martio, Nonlinear potential theory of degenerate elliptic equations, Oxford Mathematical Monographs, The Clarendon Press Oxford University Press, New York, 1993, ISBN 0-19-853669-0.

[16] V. Kokilasvili and S. Samko, Maximal and fractional operators in weighted $L^{p(x)}$ spaces, Rev. Mat. Iberoamericana (to appear).

[17] O. Kováčik and J. Rákosník, On spaces $L^{p(x)}$ and $W^{k, p(x)}$, Czechoslovak Math. J. 41(116) (1991), 592-618. 
[18] J. Malý and W. P. Ziemer, Fine regularity of solutions of elliptic partial differential equations, Mathematical Surveys and Monographs, vol. 51, American Mathematical Society, Providence, RI, 1997, ISBN 0-8218-0335-2.

[19] O. Martio and J. Sarvas, Injectivity theorems in plane and space, Ann. Acad. Sci. Fenn. Ser. A I Math. 4 (1979), 383-401.

[20] V. G. Maz'ja, Sobolev spaces, Springer Series in Soviet Mathematics, Springer-Verlag, Berlin, 1985, ISBN 3-540-13589-8.

[21] L. Pick and M. Růžička, An example of a space $L^{p(x)}$ on which the Hardy-Littlewood maximal operator is not bounded, Expo. Math. 19 (2001), 369-371.

[22] Michael Růžička, Electrorheological fluids: modeling and mathematical theory, Lecture Notes in Mathematics, vol. 1748, Springer-Verlag, Berlin, 2000, ISBN 3-540-41385-5. 